\title{
Experimental gastric endoscopic submucosal dissection: training in a porcine model
}

\author{
Panagiotis Kasapidis ${ }^{a}$, Stefanos Bassioukas $^{b}$, Georgios Mavrogenisc, Georgios Tribonias ${ }^{d}$, \\ Konstantinos Delise, Sotirios Georgopoulosf, Dimitrios Christodouloug ${ }^{\mathrm{g}}$, Theodoros Emmanouil', \\ Konstantina Paraskevai, Vassilios Panterisj, Apostolos Papaloisk, John Triantafillidis', Zacharias Tsiamoulos ${ }^{\mathrm{m}}$, \\ Takataro Fukuharan ${ }^{n}$, Yoriaki Komeda ${ }^{\circ}$, Hiroshi Kashida \\ Central Clinic, Athens, Greece; Athens Medical Center, Marousi, Greece; Mediterraneo Hospital, Athens, Greece; \\ Eugenideio Hospital, Athens, Greece; Metropolitan Hospital, Piraeus, Greece; Athens Medical Center, Faliron, Greece; \\ University of Ioannina, School of Medicine, Ioannina, Greece; Konstantopoulion General Hospital, Athens, Greece; \\ Experimental-Research Center Elpen, Athens, Greece; Iaso General Hospital, Athens, Greece; East Kent University \\ Hospitals Trust Senior Clinical Researcher, Imperial College, London St Mark's Hospital/Academic Institute, UK; \\ Kansai Medical University, Hirakata, Osaka, Japan; Ohno-Higashi, Osaka-Sayama, Kindai University, Osaka, Japan
}

\section{Abstract}

${ }^{a}$ Head, Department of Gastroenterology and Endoscopy Unit, Central Clinic, Athens, Greece (Panagiotis Kasapidis); ${ }^{\mathrm{b}}$ Athens Medical Center, Maroussi, Athens, Greece (Stefanos Bassioukas); 'Department of Endoscopy, Mediterraneo Hospital, Athens, Greece (Georgios Mavrogenis); ${ }^{\mathrm{d} D e p a r t m e n t ~ o f ~ E n d o s c o p y, ~ E u g e n i d e i o ~ H o s p i t a l, ~ A t h e n s, ~ G r e e c e ~(G e o r g i o s ~ T r i b o n i a s) ; ~}{ }^{\mathrm{D}}$ Department of Gastroenterology, Metropolitan Hospital, Piraeus, Greece (Konstantinos Delis); ' ${ }^{\mathrm{f}}$ epartment of Gastroenterology and Endoscopy Unit, Athens Medical Center, Faliron, Athens, Greece (Sotirios Georgopoulos); ${ }^{\mathrm{g}}$ Gastroenterology Department, University of Ioannina, School of Medicine, Ioannina, Greece (Dimitrios Christodoulou); ${ }^{\mathrm{h}}$ Gastroenterologist, Athens, Greece (Theodoros Emmanouil); ${ }^{\mathrm{i} D e p a r t m e n t ~ o f ~ G a s t r o e n t e r o l o g y, ~ K o n s t a n t o p o u l i o n ~ G e n e r a l ~ H o s p i t a l, ~ A t h e n s, ~ G r e e c e ~}$ (Konstantina Paraskeva); ${ }^{j}$ Gastroenterologist, Athens, Greece (Vassilios Panteris); ${ }^{k}$ Experimental-Research Center ELPEN, Athens, Greece (Apostolos

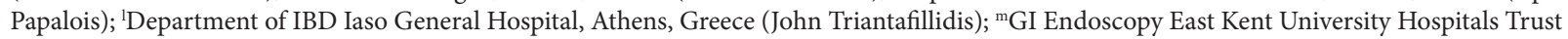
Senior Clinical Researcher, Imperial College, London St Mark's Hospital/Academic Institute, United Kingdom (Zacharias Tsiamoulos); ${ }^{\mathrm{n} D e p a r t m e n t}$ of Molecular Genetics, Institute for Biomedical Science, Division of Gastroenterology and Hepatology, The Third Department of Internal Medicine, Kansai Medical University, Hirakata, Osaka, Japan (Takataro Fukuhara); ${ }^{\circ}$ Department of Gastroenterology and Hepatology, Ohno-Higashi, OsakaSayama, Osaka, Japan, Faculty of Medicine, Kindai University (Yoriaki Komeda, Hiroshi Kashida)

Conflict of Interest: None

\begin{abstract}
Methods In the experimental research center of ELPEN Pharmaceuticals, 18 European endoscopists, inexperienced at ESD, performed gastric ESDs in porcine models. The course lasted two days and was conducted under the supervision of experts.

Results A total of 72 of 76 ESDs were completed en bloc (94.7\%). The procedural time and cutting speed differed significantly between the first and second day: $48 \pm 4.4$ vs. $43 \pm 4.8 \mathrm{~min}(\mathrm{P}=0.0045)$, and $1.38 \pm 0.20$ vs. $1.63 \pm 0.23 \mathrm{~cm}^{2} / \mathrm{min}(\mathrm{P}=0.0033)$, respectively. The complications were not nificantly different between the two groups: five (13.88\%) vs. four (11\%) episodes of bleeding acceptable rate of en bloc resections and complications.
\end{abstract}

Conclusion ESD demands a new level of endoscopic skills in Europe. A formal sequential training program, using porcine models, may benefit countries with a low volume of cases.

Keywords Endoscopic submucosal dissection, animal models, ESD training, hands-on course, procedural time

Ann Gastroenterol 2017; 30 (3): 1-4 


\section{Introduction}

Endoscopic submucosal dissection (ESD) is an established endoscopic method for the treatment of neoplasms in the gastrointestinal tract (esophagus, stomach and colorectum). ESD was developed in Japan in 1999 for the endoscopic removal of early gastric cancers [1]. Today, its use has spread worldwide. The advantages of ESD compared to other endoscopic methods (endoscopic mucosal resection) are: 1) a higher rate of complete resection; 2) superior histopathological assessment of the neoplasmatic invasion in the specimen; 3 ) decreased local recurrence; and 4) no size limitation $(\mathrm{d}>2 \mathrm{~cm})$ [2]. ESD is technically demanding and its imperfect performance may result in severe procedural complications (bleeding, perforation) and/or incomplete resection of the lesion. ESD requires special skills and a long procedure time for a quality-controlled procedure.

Although a universal training system for ESD remains to be established, some centers (firstly in the east and later in the west) are incorporating ex vivo and/or in vivo animal models to help endoscopists climb the ESD learning curve. For adequate training, the use of an animal model (in particular the stomach of a porcine model) appears to be essential before advancing to more difficult lesions, such as in the human colon [1,3-8]. To overcome these problems and facilitate the learning curve, the Endoscopy section of the Hellenic Society of Gastroenterology (ESHSG), the Hellenic Society of Gastrointestinal Oncology (HSGO) and the ExperimentalResearch Center ELPEN (E-RC) have organized (since 2014) an annual international live teaching course. This seminar (which lasts two days), provides a hands-on endoscopy course, using in vivo porcine models (swine), where the participants have the opportunity to be trained live in gastric ESD. This international training course is conducted under the auspices of the European Society for Gastrointestinal Endoscopy (ESGE) and the European Society of Digestive Oncology (ESDO).

Different devices, such as knives, and several innovations have been introduced to improve technical outcomes and to decrease the rate of procedural adverse events during ESD $[3-5,9,10]$. Despite the growing number of ESD-related publications in both asian and western countries, there is only limited evidence regarding the efficacy and value of training by means of in vivo ESD workshops $[1,5,7,8]$.

The aim of the present preliminary, prospective animal study was to compare the learning curve for gastric ESD of

Correspondence to: Panagiotis Kasapidis, MD, PhD, AGAF, Gastroenterologist, Head of Department of Gastroenterology and Endoscopy Unit, Central Clinic of Athens, Athens Greece, 49 Ymittou St., 15561 Holargos, Tel.: +30 210 3674342, Fax: +30 210 3674340, e-mail: kasapendo@yahoo.gr

Received 20 January 2017; accepted 05 March 2017; published online 12 April 2017

DOI: https://doi.org/10.20524/aog.2017.0147 individual endoscopists, during the first and the second days of the training course.

\section{Materials and methods}

The study took place in the experimental research center of ELPEN Pharmaceuticals (Athens, Greece), at a fully equipped endoscopic unit. License number 207 (16 January 2015) was issued by the Scientific Evaluation Committee of the ELPEN experimentalresearch center (European directive/legislation 63/2010). Eighteen European endoscopists, inexperienced in ESD, participated in six training sessions, for two days ( $6 \mathrm{~h} /$ day) and performed gastric ESD. Experienced tutors, from Japan and northwestern Europe, supervised the participants. One anesthetized piglet was used per station, every day. General anesthesia was administered and controlled by three veterinarian experts, who were in charge of monitoring the vital signs to prevent complications, as stipulated by regulations. The antrum and the greater curvature of the animal stomach were used for gastric ESD.

We used Olympus GIF-H180 gastroscopes during the procedures. A disposable distal transparent cap attachment (D-201-11804) was mounted onto the tip of the endoscope. The electrosurgical unit (Olympus ESG-100) was used as a power source for electrical cutting and coagulation. The Dual knife (Olympus, KD-650L), with a 2-mm tip length (non-jet injector system), was used. The settings for the knives were as follows: 1) pulse cut slow $40 \mathrm{~W}$ for mucosal incision, forced coag 1-20 W for marking the target area, and soft coagulation $80 \mathrm{~W}$ for vessel coagulation, with coag grasper (Olympus, FD411UR); and 2) forced coag 2-25 W for mucosal dissection. Sclerotherapy needles (23G) were used to inject a mixed solution (gelatin solution with slightly stained indigo carmine), for submucosal elevation.

All participants attended a 4-h theoretical session on ESD and one live demonstration of gastric ESD by an expert. Each trainee performed two gastric ESD resections per day in each specific porcine model.

Individual performance indicators (completeness of resection, procedural time and complications) were recorded on the first and second days. R0 resection was considered to have been achieved when dissection was beneath the vascular network and above the muscular layer. Endpoints were: 1) procedural time, starting from the mucosal dissection (elapsed time-cutting speed); 2) completeness of resections; and 3) complications, such as bleeding and perforation, occurring in the area of the rejected specimens.

\section{Statistical analysis}

Student's $t$-test (paired $t$-test, SPSS, version 17.0) was performed for the procedural time and resection specimens (tumor size). Chi-square test, with Yates' correction was used 




Figure 1 Gastric endoscopic submucosal dissection procedure using an in vivo porcine model. (A) Submucosal dissection is started. (B) Submucosal dissection is continued. (C) Submucosal dissection is finished. (D) The Coagrasper forceps is used to control major bleeding from large vessels

for comparison of complications between the two days. $\mathrm{P}<0.05$ was considered statistically significant.

\section{Results}

A total of 76 ESDs were performed, of which 72 (94.7\%), 4 ESDs per participant, were completed en bloc $(\mathrm{d}=3-4.5 \mathrm{~cm})$. A high en bloc R0 resection rate (93\%) was achieved during the two-day course (Fig. 1). Concerning the topography, $62.5 \%(n=45 / 72)$ of ESDs were performed in the gastric body and $37.5 \%(n=27 / 72)$ in the antrum. No statistical difference was observed between the two groups regarding the size of the tumors. The results of the procedures are summarized in Table 1.

The difference in elapsed time between the first and the second day was statistically significant: $48 \pm 4.4$ vs. $43 \pm 4.8 \mathrm{~min}$, respectively $(\mathrm{P}=0.0045)$. The cutting speed was statistically different, with 16 of 18 (88.8\%) participants improving their cutting speed during ESD on the second day of the training course: from $1.38 \pm 0.20$ to $1.63 \pm 0.23 \mathrm{~cm}^{2} / \mathrm{min}(\mathrm{P}=0.0033)$. In addition, the incidence of bleeding was higher during the first day compared to the second day, though the difference was not statistically significant-5 (13.88\%) vs. 4 episodes (11\%), $\mathrm{P}>0.05-$ and the perforation rate was similar: 2 episodes per day (5.55\%). Major bleeding (spurting), from large vessels, was treated with thermocoagulation (coag grasper). All perforations were fully clipped (Olympus long clip HX-610-135L). If any perforation occurred, an $18 \mathrm{G}$ needle was introduced into the animal abdomen to release the pressure. No mortality occurred as a result of the procedures in the animal models during the two-day course.
Table 1 Procedural data of 18 trainees performing gastric endoscopic submucosal dissection in porcine models during a two-day course

\begin{tabular}{lccc}
\hline Parameters & First day & Second day & $\mathrm{P}$ \\
\hline Operation time $(\mathrm{min})$ & $48 \pm 4.4$ & $43 \pm 4.8$ & 0.0045 \\
Cutting speed $\left(\mathrm{cm}^{2} / \mathrm{min}\right)$ & $1.38 \pm 0.20$ & $1.63 \pm 0.23$ & 0.0033 \\
Episodes of bleeding $(\mathrm{n})$ & $\mathrm{n}=5$ & $\mathrm{n}=4$ & $\mathrm{NS}$ \\
Perforation $(\mathrm{n})$ & $\mathrm{n}=2$ & $\mathrm{n}=2$ & $\mathrm{NS}$ \\
\hline
\end{tabular}

\section{Discussion}

Training in animal models is the best way to overcome the difficulties in learning ESD [1,3,4-6,8,11-13,18-21]. Concerning the type of animal model, the in vivo porcine model has proven to be the most appropriate for gastric ESD. It is recommended that ESD be performed in an in vivo porcine model, as a preliminary step before the application of ESD in humans. The general recommendation for trainees is to initiate ESD starting in the antrum (anterior and posterior wall), then progressing to the body and the fundus [5]. This type of model offers several advantages. The principal advantage of the in vivo model, as compared to the ex vivo model, is that the former is more realistic, with the presence of peristalsis, mucosal secretions and abdominal distension. Bleeding and perforation are possible real complications. The porcine model is similar to human anatomy $[4,8,13,18,19]$. Many experts on ESD advocate that at least 30 gastric ESDs should be performed in live porcine models to achieve a certain degree of mastery before attempting esophageal or colorectal ESD in humans. At the beginning of the training, it is estimated that the perforation rate may reach $20 \%[1,3,5,8,12-14]$. Our systematic training program demonstrated that novice endoscopists, under the supervision of experts, were able to perform gastric ESDs without any decline in clinical results. The overall technical performance was improved after the first day of training. We recorded acceptable rates of perforation (5.55\%) and bleeding (11-13.88\%), consistent with those in the international literature $[1,5,7,8,18,19]$. The accumulation of experience will decrease the rate of perforations. The increase in the dissection speed on the second day indicated greater acquisition of skill in the technique and therefore progress in its learning. Severe hemorrhage was not observed, because all visible vessels on the resection site were systematically coagulated after completion of the ESD. The live in vivo model provides a more realistic procedure and offers endoscopists the opportunity to respond to and to treat potential complications. Our clinical outcomes were similar with those of other recent European studies $[8,13-16,19]$. Our trainees achieved high R0 resection and procedure time rates without the occurrence of any severe complications. This suggests that our live training programs in ESD are useful for novice endoscopists. A prolonged operation time for gastric ESD in the porcine model was mostly due to the location of the lesion, the size of the lesion and the complications. The trainees who achieved faster procedural time and cutting speed started ESD in the antrum, where the location is more convenient for dissection. Furthermore, 


\section{Summary Box}

\section{What is already known:}

- Endoscopic submucosal dissection (ESD) is an established endoscopic method for the treatment of neoplasms in the gastrointestinal tract

- ESD is technically demanding and inexperience can result in severe procedural complications and/ or incomplete resection of the tumor

\section{What the new findings are:}

- In vivo porcine models are useful for improving the learning curve for ESD

- A formal hands-on sequential training program may be of most benefit in countries with a low volume of cases

the absence of major complications was beneficial for some participants as regards the operation time.

Because of the low prevalence of early gastric neoplasms, the number of patients who can benefit from ESD in Western countries is small. Hence, the interval between ESD procedures in humans can be too long, and the endoscopist's technical skills and acquired confidence can be decreased as a result. On the other hand, colorectal ESDs in western countries are more common, but are demanding procedures.

To overcome these problems in western countries, the authors of the study suggest that before colorectal ESD is performed, it must have previously been performed in an in vivo porcine model, sequentially for keeping a track record. For these reasons, the ESHSG, the HSGO and the E-RC, have organized (since 2014) an annual international live teaching hands-on course in porcine models for gastric ESD. These are in accordance with recent European position statements and guidelines on ESD [17,22]. As the number of endoscopists performing ESD and its indications continue to rise in western countries, it seems that a standardized ESD training program for teaching centers is needed.

The weakness of this study is the absence of real pathological lesions. The procedures were performed at easier, not difficult, locations (antrum and greater curvature, instead of the lesser curvature and fundus). The location is closely associated with a prolonged operation time. In addition, the stomachs of the pigs were thick with relatively small blood vessels, decreasing the risk of adverse events.

In conclusion, the use of in vivo porcine models for gastric ESD hands-on training is a safe and effective approach that could help the inexperienced endoscopists to climb the learning curve. A formal training program may be of most benefit in countries that have a low volume of cases. ESD demands a new level of endoscopic skill in Europe. To achieve safe and proficient colorectal ESD in humans, intensive learning with expert supervision is absolutely necessary.

\section{References}

1. Ohata K, Nonaka K, Misumi Y, et al. Usefulness of training using animal models for colorectal endoscopic submucosal dissection: is experience performing gastric ESD really needed? Endosc Int Open 2016;4:E333-E339.

2. Cao Y, Liao C, Tan A, Gao Y, Mo Z, Gao F. Meta-analysis of endoscopic submucosal dissection versus endoscopic mucosal resection for tumors of the gastrointestinal tract. Endoscopy 2009;41:751-757.

3. Tanaka S, Oka S, Kaneko I, et al. Endoscopic submucosal dissection for colorectal neoplasia: possibility of standardization. Gastrointest Endosc 2007;66:100-107.

4. González N, Parra-Blanco A, Villa-Gómez M, et al. Gastric endoscopic submucosal dissection: from animal model to patient. World J Gastroenterol 2013;19:8326-8334.

5. Horii J, Goto O, Shimoda M, et al. Which part of a porcine stomach is suitable as an animal training model for gastric ESD? Endoscopy 2016;48:188-193.

6. Herreros de Tejada A. ESD training: A challenging path to excellence. World J Gastrointest Endosc 2014;6:112-120.

7. Kwon CI, Kim G, Chung IK, et al. Endoscopic submucosal dissection using a novel versatile knife: An animal feasibility study (with video). Clin Endosc 2014;47:544-554.

8. Bok GH, Cho JY. ESD Hands-on course using ex vivo and in vivo models in South Korea. Clin Endosc 2012;45:358-361.

9. Conlin A, Kaltenbach T, Kusano C, Matsuda T, Oda I, Gotoda T. Endoscopic resection of gastrointestinal lesions: advancement in the application of endoscopic submucosal dissection. J Gastroenterol Hepatol 2010;25:1348-1357.

10. Oyama T. Counter traction makes endoscopic submucosal dissection easier. Clin Endosc 2012;45:375-378.

11. Saito Y, Otake Y, Sakamoto T, et al. Indications for and technical aspects of colorectal endoscopic submucosal dissection. Gut Liver 2013;7:263-269.

12. Gotoda T, Friedland S, Hamanaka H, Soetikno R. A learning curve for advanced endoscopic resection. Gastrointest Endosc 2005;62:866-867.

13. Berr F, Ponchon T, Neureiter D, et al. Experimental endoscopic submucosal dissection training in a porcine model: learning experience of skilled Western endoscopists. Dig Endosc 2011;23:281-289.

14. Pioche M, Ciocirlan M, Lépilliez V, et al. High-pressure jet injection of viscous solutions for endoscopic submucosal dissection: a study on ex vivo pig stomachs. Surg Endosc 2014;28:1742-1747.

15. Ribeiro-Mourão F, Pimentel-Nunes P, Dinis-Ribeiro M. Endoscopic submucosal dissection for gastric lesions: results of an European inquiry. Endoscopy 2010;42:814-819.

16. Farhat S, Chaussade S, Ponchon T, et al; SFED ESD study group. Endoscopic submucosal dissection in a European setting. A multiinstitutional report of a technique in development. Endoscopy 2011;43:664-670.

17. Deprez PH, Bergman JJ, Meisner S, et al. Current practice with endoscopic submucosal dissection in Europe: position statement from a panel of experts. Endoscopy 2010;42:853-858.

18. Draganov PV, Chang M, Coman RM, Wagh MS, An Q, Gotoda T. Role of observation of live cases done by Japanese experts in the acquisition of ESD skills by a western endoscopist. World $J$ Gastroenterol 2014;20:4675-4680.

19. Parra-Blanco A, Arnau MR, Nicolás-Pérez D, et al. Endoscopic submucosal dissection training with pig models in a Western country. World J Gastroenterol 2010;16:2895-2900.

20. Zizer E, Roppenecker D, Helmes F, et al. A new 3D-printed overtube system for endoscopic submucosal dissection: first results of a randomized study in a porcine model. Endoscopy 2016;48:762-765.

21. Mavrogenis G, Hochberger J, Deprez P, Shafazand M, Coumaros D, Yamamoto K. Technological review on ESD: available equipment, recent developments and emerging techniques. Scand J Gastroenterol 2017;52:486-498.

22. Pimentel-Nunes P, Dinis-Ribeiro M, Ponchon T, et al. Endoscopic submucosal dissection: European Society of Gastrointestinal Endoscopy (ESGE) Guideline. Endoscopy 2015;47:829-854. 